\title{
A precise analytical approximation for the deprojection of the Sérsic profile
}

\author{
Eduardo Vitral and Gary A. Mamon
}

\begin{abstract}
Institut d'Astrophysique de Paris (UMR 7095: CNRS \& Sorbonne Université), 98 Bis Bd Arago, 75014 Paris, France e-mail: vitral@iap.fr
\end{abstract}

Received 27 November 2019 / Accepted 8 January 2020

\begin{abstract}
The Sérsic model shows a close fit to the surface brightness (or surface density) profiles of elliptical galaxies and galaxy bulges, and possibly also those of dwarf spheroidal galaxies and globular clusters. The deprojected density and mass profiles are important for many astrophysical applications, in particular for mass-orbit modeling of these systems. However, the exact deprojection formula for the Sérsic model employs special functions that are not available in most computer languages. We show that all previous analytical approximations to the $3 \mathrm{D}$ density profile are imprecise at low Sérsic index $(n \lesssim 1.5)$. We derived a more precise analytical approximation to the deprojected Sérsic density and mass profiles by fitting two-dimensional tenth-order polynomials to the residuals of the analytical approximations by Lima Neto et al. (1999, MNRAS, 309, 481; LGM) for these profiles, relative to the numerical estimates. Our LGM-based polynomial fits have typical relative precision better than $0.2 \%$ for both density and mass profiles, for Sérsic indices $0.5 \leq n \leq 10$ and radii $0.001<r / R_{\mathrm{e}}<1000$. Our approximation is much more precise than previously published approximations (except, in some models, for a few discrete values of the index). An appendix compares the deprojected Sérsic profiles with those of other popular simple models.
\end{abstract}

Key words. methods: numerical - galaxies: elliptical and lenticular, cD - galaxies: structure - globular clusters: general galaxies: bulges

\section{Introduction}

The Sérsic model (Sérsic 1963; Sersic 1968) is the generalization of the $R^{1 / 4}$ law (de Vaucouleurs 1948) to describe the surface brightness profiles of elliptical galaxies (Caon et al. 1993) and the bulges of spiral galaxies (Andredakis et al. 1995). It has also been used to describe the surface density profiles of nuclear star clusters (Graham \& Spitler 2009), resolved dwarf spheroidal galaxies (Battaglia et al. 2006), and globular clusters (Barmby et al. 2007).

The surface (mass or number) density (or equivalently surface brightness) of the Sérsic model is

$\Sigma(R)=\Sigma_{0} \exp \left[-b_{n}\left(\frac{R}{R_{\mathrm{e}}}\right)^{1 / n}\right]$,

where $R$ is the projected distance to the source center, $R_{\mathrm{e}}$ is the effective radius containing half of the projected luminosity, $n$ is the Sérsic index, and $\Sigma_{0}$ is the central surface density. The term $b_{n}$ is a function of $n$, obtained by solving the equation:

$\Gamma(2 n) / 2=\gamma\left(2 n, b_{n}\right)$,

where $\gamma(a, x)=\int_{0}^{x} t^{a-1} e^{-t} \mathrm{~d} t$ is the lower incomplete gamma function.

Since the Sérsic model accurately represents astronomical objects viewed in projection, it is important to know its corresponding three-dimensional (3D) density and mass profiles. These serve as a reference for comparison with other possible observational tracers, as well as to dark matter. Moreover, the 3D density profile is required for modeling the kinematics of spherical structures because it appears in the Jeans equation of local dynamical equilibrium. Since the Jeans equation also contains the total mass profile, the 3D mass profiles of stellar components are required to estimate the dark matter mass profile of elliptical and dwarf spheroidal galaxies.

Many authors assume that simple three-dimensional models resemble Sérsic models for certain values of the Sérsic index: It is often assumed that massive ellipticals and spiral bulges are well represented by the Hernquist (1990) model (e.g., Widrow \& Dubinski 2005). On the other hand, dwarf spheroidal galaxies are often described with the Plummer (1911) model (e.g., Muñoz et al. 2018, who also tried Sérsic and other models), while ultra diffuse galaxies have been described with the Einasto (Einasto 1965; Navarro et al. 2004) ${ }^{1}$ model (Nusser 2019). Łokas \& Mamon (2001) noted that the projected Navarro et al. (1996, hereafter NFW) model resembles an $n=3$ Sérsic for reasonable concentrations. Finally, $n=4$ Sérsic models are considered to resemble the Jaffe (1983) model (Ciotti et al. 2019). In Appendix A, we compare these models to the deprojected Sérsic.

Unfortunately, the deprojection of the Sérsic surface density profile to a $3 \mathrm{D}$ (mass or number) ${ }^{2}$ density profile, through Abel (1826) inversion

$\rho(r)=-\frac{1}{\pi} \int_{r}^{+\infty} \frac{\mathrm{d} \Sigma}{\mathrm{d} R} \frac{\mathrm{d} R}{\sqrt{R^{2}-r^{2}}}$,

\footnotetext{
1 Navarro et al. (2004) showed how the Einasto model accurately represents the density profiles of dark matter halos in dissipationless cosmological simulations, while Merritt et al. (2005) were the first to note its similar form to the Sérsic model, and Merritt et al. (2006) were first to realize that this model had been previously introduced by Einasto.

2 The number profile always has the same form as the mass profile, and is obtained by simply replacing $M(r)$ with $N(r)$ and $M_{\infty}$ with $N_{\infty}$, e.g., in Eqs. (7), (8), and (17).
} 
(e.g., Binney \& Mamon 1982), as well as the corresponding 3D mass (or number) profile

$M(r)=\int_{0}^{r} 4 \pi s^{2} \rho(s) \mathrm{d} s$,

both involve the complicated Meijer G special function (Mazure \& Capelato 2002 for integer values of $n$, and Baes \& Gentile 2011 for general values of $n$ ) or the other, complicated Fox $H$ function (Baes \& van Hese 2011), neither of which are available in popular computer languages.

Following the shape of the analytical approximation to the $R^{1 / 4}$ law by Mellier \& Mathez (1987), Prugniel \& Simien (1997, hereafter, PS) proposed an analytical approximation for the $3 \mathrm{D}$ density of the Sérsic profile:

$\rho_{\mathrm{PS}}(r)=\rho_{0}\left(\frac{r}{R_{\mathrm{e}}}\right)^{-p_{n}} \exp \left[-b_{n}\left(\frac{r}{R_{\mathrm{e}}}\right)^{1 / n}\right]$,

where $p_{n}$ is a function depending only on $n$ :

$p_{n, \mathrm{PS}}=1-\frac{0.594}{n}+\frac{0.055}{n^{2}}$.

Equation (5) yields a simple analytical form for the 3D mass profile,

$M_{\mathrm{PS}}(r)=M_{\infty} \frac{\gamma\left[\left(3-p_{n}\right) n, b_{n}\left(r / R_{\mathrm{e}}\right)^{1 / n}\right]}{\Gamma\left[\left(3-p_{n}\right) n\right]}$,

$M_{\infty}=4 \pi \rho_{0} R_{\mathrm{e}}^{3} \frac{n \Gamma\left[\left(3-p_{n}\right) n\right]}{b_{n}^{\left(3-p_{n}\right) n}}$,

Lima Neto et al. (1999, hereafter LGM) later perfected the approximation of Eq. (6) with

$p_{n, \mathrm{LGM}}=1-\frac{0.6097}{n}+\frac{0.05463}{n^{2}}$.

According to LGM, Eq. (9) has 5\% relative accuracy for $0.56 \leq$ $n \leq 10$ and $-2<\log \left(r / R_{\mathrm{e}}\right)<3$. However, the power-law approximation at small radii is unjustified for small $n$. Indeed, as shown by Baes \& Gentile (2011), the central density profile converges to a finite value for $n<1$ (and the inner density profile diverges only logarithmically for $n=1$ ), as we illustrate in Sect. 3.

Simonneau \& Prada (1999, 2004, hereafter SP) proposed the quasi-Gaussian expansion for the density profile

$\rho_{\mathrm{SP}}(r)=\frac{2}{\pi} \frac{b_{n}}{(n-1)} \frac{\Sigma_{0}}{R_{\mathrm{e}}}\left(\frac{r}{R_{\mathrm{e}}}\right)^{1 / n-1} \sum_{j=1}^{5} \rho_{j} \exp \left[-b_{n} \lambda_{j}\left(\frac{r}{R_{\mathrm{e}}}\right)^{1 / n}\right]$,

where

$\lambda_{j}=\left(1-x_{j}^{2}\right)^{-1 /(n-1)}$,

$\rho_{j}=w_{j} \frac{x_{j}}{\sqrt{1-\left(1-x_{j}^{2}\right)^{2 n /(n-1)}}}$,

where $x_{j}$ and $w_{j}$ are ten fit parameters. The individual SP density profiles (the terms inside the sum of Eq. (10)) have a similar (but not the same) form to that of the PS/LGM one, hence the similar shape of the mass profile:

$$
\begin{aligned}
M_{\mathrm{SP}}(r)= & M_{\infty} \frac{4}{\pi(n-1) \Gamma(2 n)} \\
& \times \sum_{j=1}^{5} \frac{\rho_{j}}{\lambda_{j}^{2 n+1}} \gamma\left[2 n+1, b_{n} \lambda_{j}\left(\frac{r}{R_{\mathrm{e}}}\right)^{1 / n}\right] .
\end{aligned}
$$

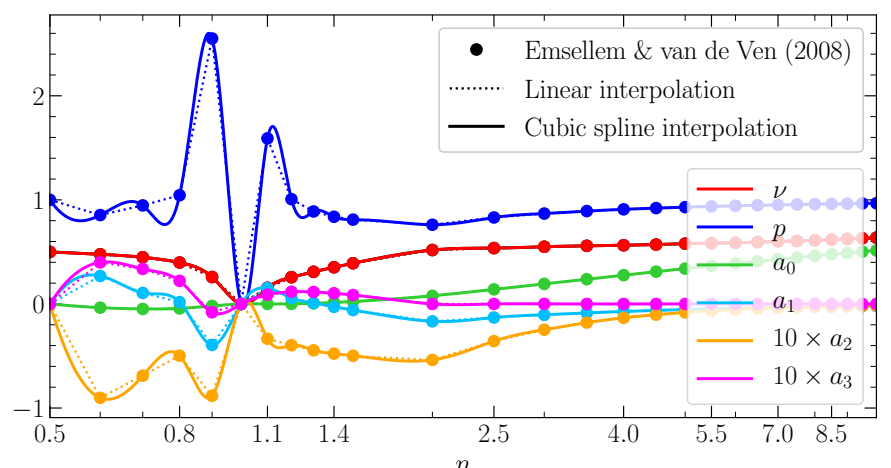

Fig. 1. Variation with Sérsic index of the different parameters of the analytical approximation of Emsellem \& van de Ven (2008) for the deprojected Sérsic density profile (filled circles). The solid and dotted curves show the spline cubic and linear interpolations, respectively. At small $n$, the parameters vary abruptly and the interpolations (both linear and cubic) are therefore uncertain.

Trujillo et al. (2002) proposed an ellipsoid formula, which in the limit of spherical symmetry becomes

$\rho_{\mathrm{T}}(r)=\frac{2^{(n-1) /(2 n)} b_{n}}{\pi n} \frac{\Sigma_{0}}{R_{\mathrm{e}}} r^{p_{n}(1 / n-1)} \frac{K_{v_{n}}\left(r / R_{\mathrm{e}}\right)}{1-\sum_{i=0}^{2} a_{n, i} \log ^{i}\left(r / R_{\mathrm{e}}\right)}$,

where $K_{v}(x)$ is the modified Bessel function of the second kind ${ }^{3}$ of index $v$, while $v_{n}, p_{n}, a_{n, 0}, a_{n, 1}$, and $a_{n, 2}$ are empirical functions of index $n$. Trujillo et al. (2002) only provided their results for integer and half-integer values of $n$ for $n \leq 5$ and only integer values of $n$ beyond. Emsellem \& van de Ven (2008, hereafter EV) repeated their analysis on a finer grid of $n$, with steps of 0.1 for $0.5 \leq n \leq 1.5$ and with one more term, $a_{n, 3}$, in Eq. (14) involving 168 parameters. However, as shown in Fig. 1, these functions vary abruptly for $n \lesssim 1.2$. Moreover, neither Trujillo et al. (2002) nor Emsellem \& van de Ven (2008) provide analytical forms for the mass profile.

In summary, all the previous approximations to the deprojected Sérsic model have drawbacks:

- Both PS and LGM (and Márquez et al. 2000, which is the same as LGM, but with a slightly different last term for $p_{n}$, which was a typo) are inappropriate for low $n$ (Baes \& Gentile 2011) and less precise than claimed (Emsellem \& van de Ven 2008).

- SP is limited to $n \geq 1$, and is generally less precise than EV.

- Trujillo et al. (2002) is only given for half-integer values of $n$ and their parameters vary wildly with $n$ for $n \leq 1.5$. These authors do not provide a formula for the mass profile.

- EV also suffers from discrete values of $n$, even though the grid is finer $(\Delta n=0.1$ for $n \leq 1.5)$. These authors also did not provide a formula for the mass profile.

In this article, we provide polynomial fits to the log residuals of the LGM approximation, which allow high accuracy to be reached for both the $3 \mathrm{D}$ density and $3 \mathrm{D}$ mass profiles in a wide range of Sérsic indices. In Sect. 2, we present the mathematical formalism and briefly explain our numerical integration method. We then show in Sect. 3 how our polynomial plus LGM approximation is orders of magnitude more precise than the formulae of LGM, SP, and Trujillo et al. (2002), as well as that of EV for low $n$, and is only slightly less precise for $n \gtrsim 3$. We conclude and discuss our results in Sect. 4.

3 Trujillo et al. (2002) call this the modified Bessel function of the third kind, as some others do. 


\section{Method}

\subsection{Equations using dimensionless profiles}

We express the general surface density, 3D density, and 3D mass (or number) profiles in terms of dimensionless functions:

$\Sigma(R)=\frac{M_{\infty}}{\pi R_{\mathrm{e}}^{2}} \widetilde{\Sigma}\left(\frac{R}{R_{\mathrm{e}}}\right)$,

$\rho(r)=\left(\frac{M_{\infty}}{4 \pi R_{\mathrm{e}}^{3}}\right) \widetilde{\rho}\left(\frac{r}{R_{\mathrm{e}}}\right)$,

$M(r)=M_{\infty} \widetilde{M}\left(\frac{r}{R_{\mathrm{e}}}\right)$.

Hereafter, we use $x=r / R_{\mathrm{e}}$ and $X=R / R_{\mathrm{e}}$. For the Sérsic model (see Graham \& Driver 2005 for a thorough review of the Sérsic profile), the dimensionless surface density profile is

$\widetilde{\Sigma}_{\mathrm{S}}(X)=\frac{b_{n}^{2 n}}{2 n \Gamma(2 n)} \exp \left(-b_{n} X^{1 / n}\right)$

while for the PS model, one can write the dimensionless 3D density and mass profiles as

$$
\begin{aligned}
\widetilde{\rho}_{\mathrm{PS}}(x) & =\frac{b_{n}^{\left(3-p_{n}\right) n}}{n \Gamma\left[\left(3-p_{n}\right) n\right]} x^{-p_{n}} \exp \left[-b_{n} x^{1 / n}\right], \\
\widetilde{M}_{\mathrm{PS}}(x) & =\frac{\gamma\left[\left(3-p_{n}\right) n, b_{n} X^{1 / n}\right]}{\Gamma\left(\left(3-p_{n}\right) n\right)} .
\end{aligned}
$$

It is easy to show that the deprojection Eq. (3) becomes

$\widetilde{\rho}(x)=-\frac{4}{\pi} \int_{x}^{+\infty} \frac{\mathrm{d} \widetilde{\Sigma}}{\mathrm{d} X} \frac{\mathrm{d} X}{\sqrt{X^{2}-x^{2}}}$,

where

$\frac{\mathrm{d} \widetilde{\Sigma}}{\mathrm{d} X} \equiv \widetilde{\Sigma}^{\prime}(X)=-\frac{b_{n}^{2 n+1}}{2 n^{2} \Gamma(2 n)} X^{-1+1 / n} \exp \left(-b_{n} x^{1 / n}\right)$.

The dimensionless mass profile is

$$
\begin{aligned}
\widetilde{M}(x)= & \int_{0}^{x} y^{2} \widetilde{\rho}(y) \mathrm{d} y=-\frac{4}{\pi} \int_{0}^{x} y^{2} \mathrm{~d} y \int_{y}^{\infty} \frac{\widetilde{\Sigma}^{\prime}(X)}{\sqrt{X^{2}-y^{2}}} \mathrm{~d} X \\
= & -\int_{0}^{x} X^{2} \widetilde{\Sigma}^{\prime}(X) \mathrm{d} X-\frac{2}{\pi} \int_{x}^{\infty} X^{2} \sin ^{-1}\left(\frac{x}{X}\right) \widetilde{\Sigma}^{\prime}(X) \mathrm{d} X \\
& +\frac{2}{\pi} \int_{x}^{\infty} x \sqrt{X^{2}-x^{2}} \widetilde{\Sigma}^{\prime}(X) \mathrm{d} X,
\end{aligned}
$$

where Eq. (24) is obtained by inversion of the order of integration in the second equality of Eq. (23).

\subsection{Numerical integration}

We numerically evaluated the dimensionless 3D density (Eq. (21)) and mass (Eq. (24)) profiles by performing the numerical integrations in cells $50 \times 100$ of $\left[\log n, \log \left(r / R_{\mathrm{e}}\right)\right]$, with $\log 0.5 \leq \log n \leq 1$ and $-3 \leq \log \left(r / R_{\mathrm{e}}\right) \leq 3$. Numerical calculations were done with Python's SCIPY.INTEGRATE.QUAD. For both density and mass profiles, we split the numerical integration in two, that is,

$\int_{a}^{b} f(X) \mathrm{d} X=\int_{a}^{X_{\text {crit }}} f(X) \mathrm{d} X+\int_{X_{\text {crit }}}^{b} f(X) \mathrm{d} X$,

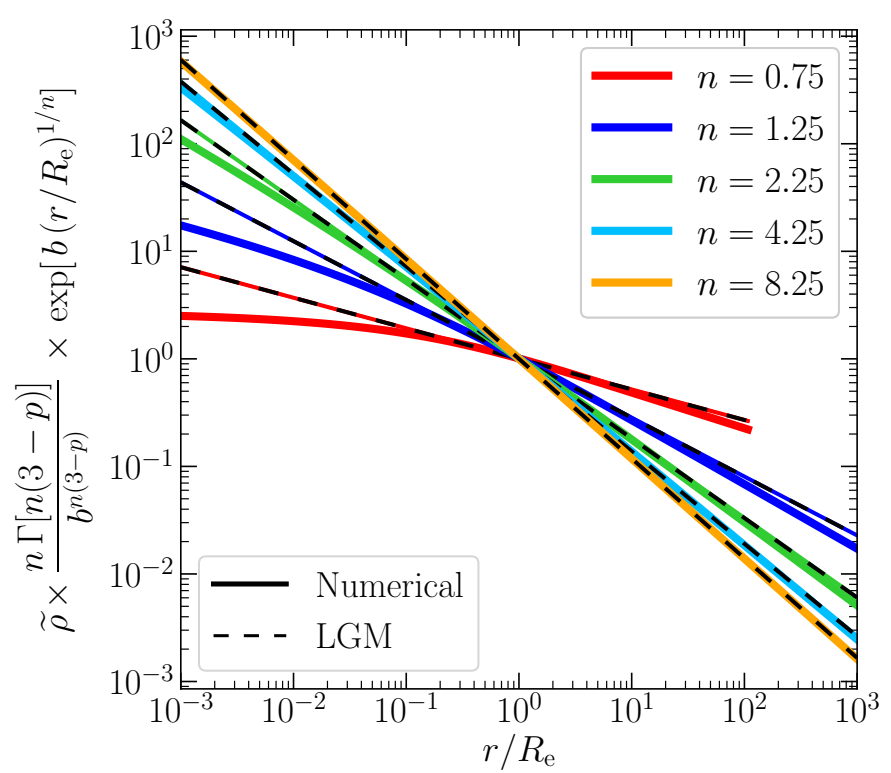

Fig. 2. Illustration of the accuracy of the PS formula with the LGM coefficients for $p_{n}$. The solid curves show the numerically estimated profiles, while the colored-dashed curves show the LGM approximation.

where $\exp \left(-b_{n} X_{\text {crit }}^{1 / n}\right)=10^{-9}$ and $a \leq X_{\text {crit }} \leq b$. We used a relative tolerance of epsrel $=10^{-4}$ and limit $=1000$ in both integrals. If $X_{\text {crit }} \notin[a, b]$, we also used epsrel $=10^{-4}$ and limit $=1000$, but for a single integral from $a$ to $b$.

We performed our analysis using either the highly accurate approximations for $b_{n}$ of Ciotti \& Bertin (1999, hereafter, CB) or the exact (numerical) solutions of Eq. (2). We noticed that the difference between these two approaches was negligible (see Sect. 3).

We then fit two-dimensional polynomials to both $\log \left[\widetilde{\rho}_{\mathrm{LGM}}(x, n) / \widetilde{\rho}(x, n)\right]$ and $\log \left[\widetilde{M}_{\mathrm{LGM}}(x, n) / \widetilde{M}(x, n)\right]$, for geometrically spaced $x$ and $n$, writing

$\log \left[\widetilde{f}_{\mathrm{LGM}} / \widetilde{f}\right]=-\sum_{i=0}^{k} \sum_{j=0}^{k-i} a_{i j} \log ^{i} x \log ^{j} n$,

with polynomial orders $2 \leq k \leq 12$. For this, we used Python's package NUMPY.LINALG.LSTSQ. We found the smallest residuals for tenth-order polynomials when using both the $b_{n}$ approximation of $\mathrm{CB}$ and $b_{n}$ by numerically solving Eq. (2). The coefficients are provided in Tables B.1-B.4. In the rest of the paper, we present the results relative to the $\mathrm{CB}$ approximation, since it is a simpler and more used model, and also because our tenthorder polynomial fits the exact $b_{n}$ case remarkably well.

\subsection{Numerical precision: tests for known simple analytical deprojections $(n=0.5$ and 1$)$}

For Sérsic indices $n=0.5$ and $n=1$, there are analytical solutions for the 3D density profile:

$\widetilde{\rho}(x)= \begin{cases}4 \frac{b_{0.5}^{3 / 2}}{\sqrt{\pi}} \exp \left[-b_{0.5} x^{2}\right] & (n=0.5), \\ 2 \frac{b_{1}^{3}}{\pi} K_{0}\left(b_{1} x\right) & (n=1),\end{cases}$ 

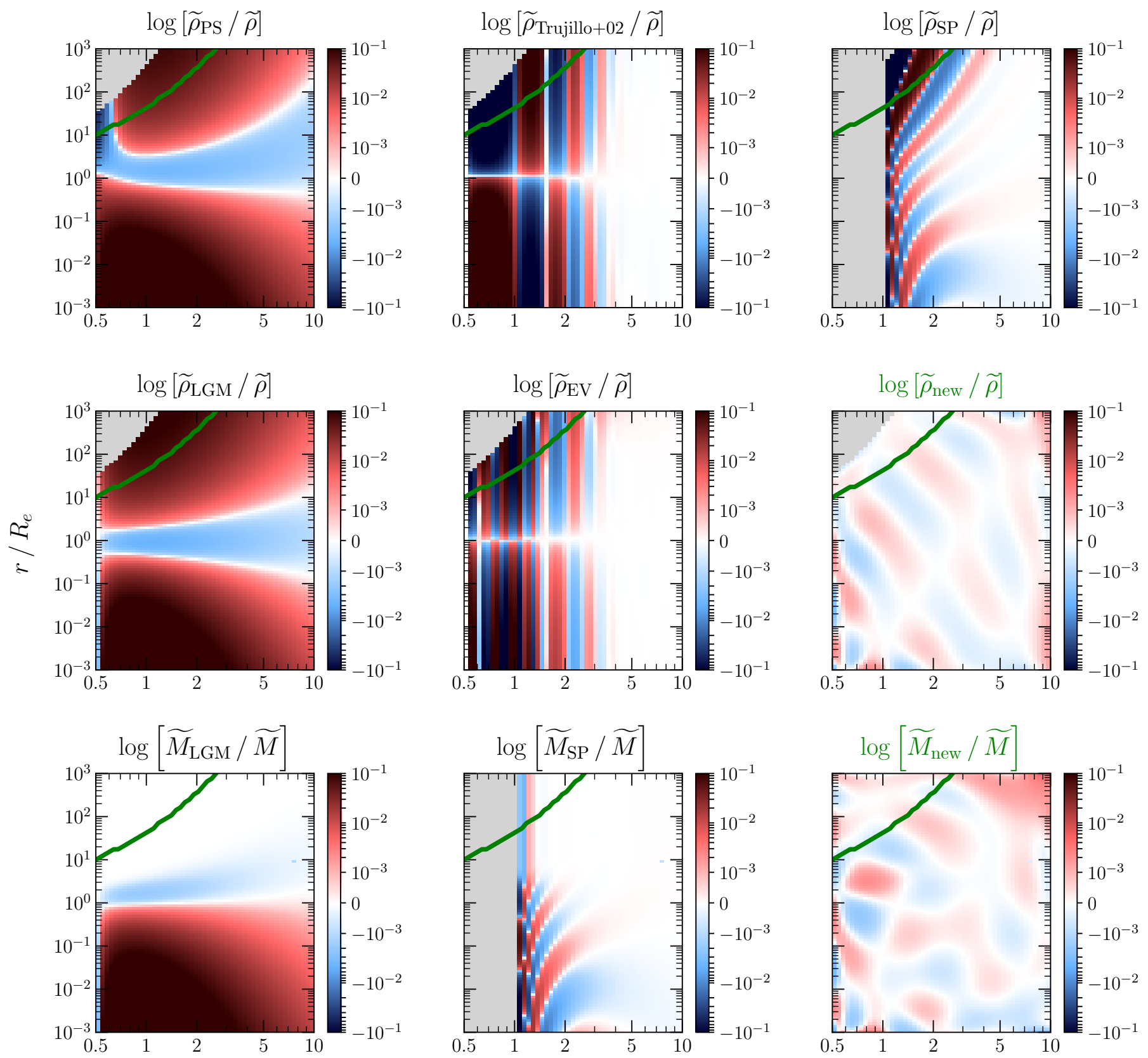

$n$

Fig. 3. Accuracy of deprojected density (top 6 panels) and mass ( 3 bottom panels) of the different analytical approximations (PS: Prugniel \& Simien 1997; LGM: Lima Neto et al. 1999; SP: Simonneau \& Prada 1999, 2004; Trujillo+02: Trujillo et al. 2002; EV: Emsellem \& van de Ven 2008) and our new one (Eq. (28), with green-colored titles) as a function of both Sérsic index (abscissae) and radius (ordinates). The color scale given in the vertical color bars is linear for log ratios between -0.001 and 0.001 and logarithmic beyond. The gray region and green curves in the upper left of the density panels are for regions where the numerical integration reached the underflow limit or density $10^{-30}$ times $\rho\left(R_{\mathrm{e}}\right)$, respectively, because of the very rapid decline of density at large radii for low $n$, and also covers $n<1$, which is not covered by the SP model. We note that the EV and Trujillo+02 models perform better at specific values of $n$ that are often missed in our grid.

where $K_{0}(x)$ is the modified Bessel function of the second kind of index 0 . We can therefore verify the numerical integration of Eq. (21) for these two Sérsic indices.

For the interval $-3 \leq \log \left(r / R_{\mathrm{e}}\right) \leq 3$, we compared the densities from numerical integration with the analytical formulae of Eq. (27) using the CB approximation for $b_{n}$. The match is very good, with root-mean-square (rms) values of $\log \left(\widetilde{\rho}_{\text {ana }} / \widetilde{\rho}_{\text {num }}\right)$ of $1.5 \times 10^{-7}$ and $2 \times 10^{-8}$ for $n=0.5$ and $n=1$, respectively. The same comparison using the exact $b_{n}$ yields $7 \times 10^{-5}$ and $2 \times 10^{-8}$, respectively (with one particular value of $r$ causing the higher rms for $n=0.5)$.

\section{Results}

As seen in Fig. 2, the 3D density profiles depart from the power laws proposed by LGM at low $n$, especially for low radii, as expected by the asymptotic expansions of Baes \& Gentile (2011) for $n<1$. Interestingly, the LGM formula is also inadequate at 


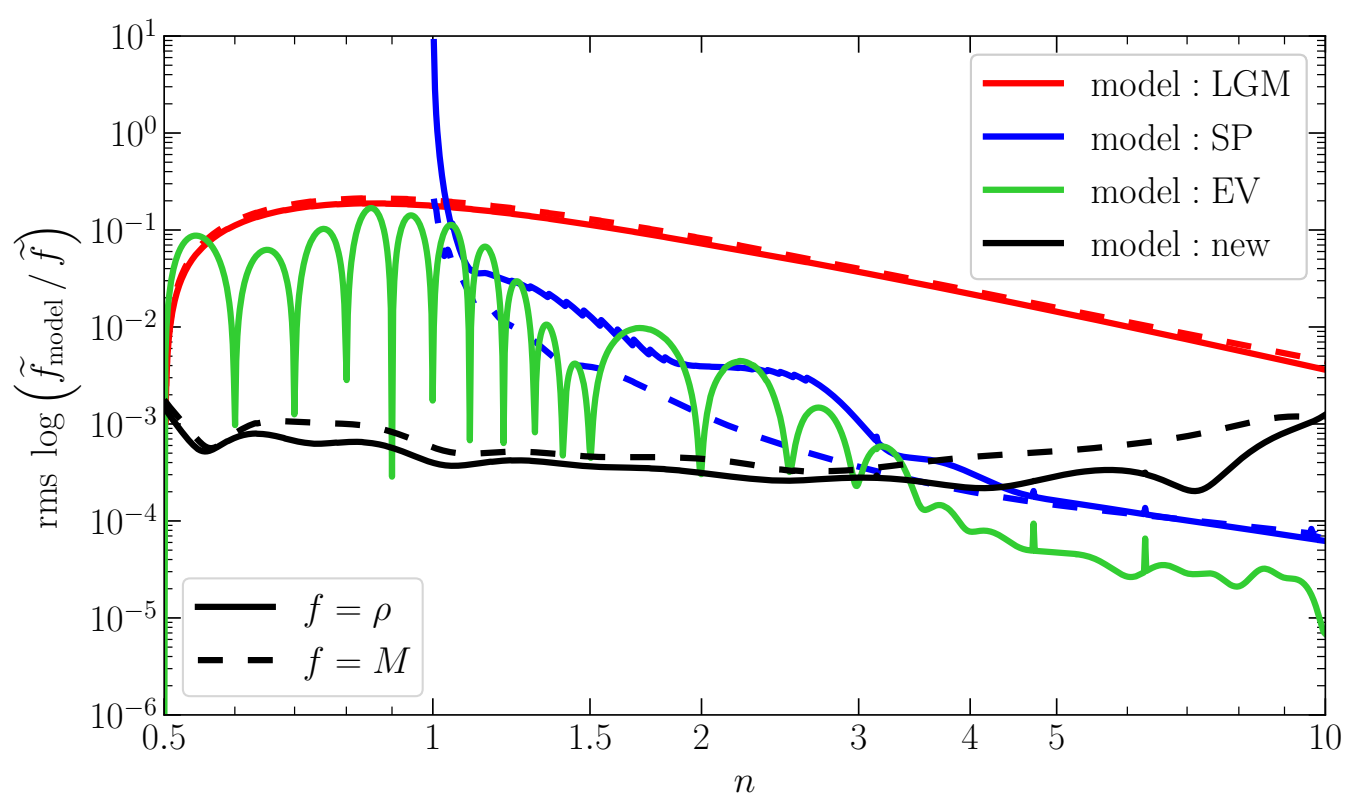

Fig. 4. Accuracy of different approximations (LGM: Lima Neto et al. 1999; SP: Simonneau \& Prada 1999, 2004; EV: Emsellem \& van de Ven 2008) and our new one (Eq. (28)) as a function of Sérsic index. We note that the EV model performs better at specific values of $n$ that are often missed in our logarithmic grid of 1000 values of $n$.

low radii for $n=1.25$ and 2.25 , although the asymptotic expansion of Baes \& Gentile (2011) indicate power-law behavior at small radii. This poor accuracy of the LGM approximation at low radii is a serious concern when performing kinematic modeling of systems with possible central massive black holes. For example, Gaia second data release (DR2) positions and proper motions for stars in nearby globular clusters extend inwards to 0.7 arcsec from the center, which translates to $0.002 R_{\mathrm{e}}$.

We now compare the accuracy of the different analytical approximations for the 3D density and 3D mass profiles. Figure 3 displays the ratio $\log \left(\widetilde{f}_{\text {model }} / \widetilde{f}\right)$, for $\widetilde{f}=\widetilde{\rho}$ and $\widetilde{f}=\widetilde{M}$, for the main analytical approximations available in the literature, along with our new model

$f_{\text {new }}(x, n)=f_{\mathrm{LGM}}(x, n) \operatorname{dex}\left[\sum_{i=0}^{10} \sum_{j=0}^{10-i} a_{i, j} \log ^{i} x \log ^{j} n\right]$,

where $f$ is either the $3 \mathrm{D}$ density or 3D mass profile. We see that our model presents a more continuous behavior over the full range of Sérsic indices and radii. Our approximation displays the smallest residuals among all models for $n \lesssim 3$ (except that SP outperforms our model for mass estimates at $r>3 R_{\mathrm{e}}$ for $n>1.3)$.

The variation of accuracy with Sérsic index can be seen in more detail in Fig. 4, which displays the rms of $\log \left(\widetilde{f}_{\text {model }} / \widetilde{f}\right)$, over the radial domain where $\rho(r)>10^{-30} \rho\left(R_{\mathrm{e}}\right)$, of the main analytical approximations using $1000 \log$-spaced Sérsic indices. Figure 4 indicates that the SP and EV approximations for density are less accurate than $2.3 \%\left(0.01\right.$ for $\left.\log \left(\widetilde{f}_{\text {model }} / \widetilde{f}\right)\right)$ for $n<1.6$ and $n<1.3$, respectively. Our approximation (Eq. (28)) is more accurate than SP for $n<4.3$ (density) and $n<3.1$ (mass), and is more accurate than EV for $n<3.4$ (density), except for their particular choices of $n$. Figure 4 shows that the EV approximation is much more accurate at specific values of $n$ (we note that our grid does not contain all of these values precisely, and therefore the EV approximation is even more accurate at these specific values of $n$ ). However, these specific values of $n$ represent a negligible measure compared to the full continuous range of $0.5 \leq n \leq 10$. Therefore, the EV approximation at low $n$ is not reliable for estimating the 3D density profile.

We analyzed the results shown in Fig. 4 using $b_{n}$ from CB or by numerically solving Eq. (2), and the results were very similar. In fact, the results are similar if we adopt one form of $b_{n}$ in the numerical integration and the other in the analytical approximations. This can be explained by the fact that $\log \left(\widetilde{f}_{\mathrm{LGM}} / \widetilde{f}\right)$ is practically the same for both estimates of $b_{n}$, yielding a very similar fit of Eq. (26).

Finally, Table 1 provides the rms accuracies computed over the full range of radii $-3 \leq \log \left(r / R_{\mathrm{e}}\right) \leq 3$ and $0.5 \leq n \leq 10$, except for the SP formula, which does not allow $n \leq 1$, and also avoiding the domain where $\rho(r)<10^{-30} \rho\left(R_{\mathrm{e}}\right)$. We see that, averaging over all Sérsic indices, our approximation is much more accurate than all others (with over ten times lower rms).

\section{Conclusions and discussion}

The Sérsic model is usually considered to provide excellent fits to the surface density (or surface brightness) profiles of elliptical galaxies, spiral bulges, and even dwarf spheroidal galaxies and globular clusters. In the past, many authors have used simple analytical models to describe these systems, arguing that their models, once projected, resemble Sérsic models. It is more relevant to compare the physically meaningful three-dimensional density profiles of these simple models to the deprojected Sérsic model.

This comparison is made in Appendix A for the Plummer, Jaffe, Hernquist, Einasto, and NFW models. As seen in Fig. A.1, most of the simple models do not provide good fits to the deprojected Sérsic model, even for narrow ranges of the Sérsic index. The Plummer model requires a low index at small radii, but a much higher index at large radii, and the normalized density profile fits poorly at most radii. The Hernquist model resembles the $n=2.8$ deprojected Sérsic model at low radii and the $n=5.7$ Sérsic at large radii. The NFW models resemble the $n=2.8$ deprojected Sérsic at low radii (consistent with the 
Table 1. Accuracy of approximations to 3D density and mass profiles.

\begin{tabular}{|c|c|c|}
\hline Author & $\begin{array}{c}\mathrm{rms} \\
\log \left(\frac{\widetilde{\rho}_{\text {approx }}}{\widetilde{\rho}_{\text {num }}}\right)\end{array}$ & $\log \left(\frac{\widetilde{M}_{\text {approx }}}{\widetilde{M}_{\text {num }}}\right.$ \\
\hline Prugniel \& Simien (1997) & 0.1052 & 0.1187 \\
\hline Lima Neto et al. (1999) & 0.0905 & 0.1021 \\
\hline Simonneau \& Prada (2004) $(n>1)$ & 0.0238 & 0.0098 \\
\hline Trujillo et al. (2002) & 0.1496 & - \\
\hline Emsellem \& van de Ven (2008) & 0.0382 & - \\
\hline New & 0.0005 & 0.0007 \\
\hline Hybrid-1 (optimized for $n_{\text {cut }}$ ) & 0.0004 & 0.0005 \\
\hline Hybrid-2 (optimized for $r_{\text {cut }}$ ) & 0.0004 & 0.0005 \\
\hline
\end{tabular}

Notes. The rms accuracies are computed over the full range of radii $-3 \leq \log \left(r / R_{\mathrm{e}}\right) \leq 3$ (100 steps) and $0.5 \leq n \leq 10$ (50 steps), except for the SP formula, which does not allow $n \leq 1$, and also avoiding the domain where $\rho(r)<10^{-30} \rho\left(R_{\mathrm{e}}\right)$. Trujillo et al. (2002) and EV do not provide analytical mass profiles. The lower two rows display hybrid models, both with our new approximation $\widetilde{\rho}_{\text {new }}$ for $n<3.4$ and $\widetilde{\rho}_{\mathrm{EV}}$ for $n \geq 3.4$. The first hybrid model has a mass profile $\widetilde{M}_{\text {new }}$ for $n<3$ and $\widetilde{M}_{\mathrm{SP}}$ for $n \geq 3$, while in hybrid model 2, the mass profile is $\widetilde{M}_{\text {new }}$ for $r<R_{\mathrm{e}}$ and $\widetilde{M}_{\mathrm{LGM}}$ for $r \geq R_{\mathrm{e}}$. The values in bold highlight the accuracies with our approximations.

similarity of the projected Sérsic with NFW discovered by Łokas \& Mamon 2001), but have a shallower slope at large radii than even the shallowest $(n=8)$ deprojected Sérsic model. On the other hand, the Jaffe model resembles the $n=5.7$ model at all radii. Moreover, as seen in Fig. A.2, the Einasto model provides a fair representation (rms difference of density profiles normalized to value at half-mass radius less than $0.1 \mathrm{dex}$ ) of the deprojected Sérsic model for $n>6.5$.

We reconsidered the different analytical deprojections of the Sérsic surface brightness (or surface density) profile. We found that the analytical approximations present in the literature do not show satisfying results when the Sérsic index is in the range $0.5 \leq n \lesssim 1.5$ (apart from the specific values of $n$ given by Emsellem \& van de Ven 2008). In particular, the power-law times exponential density profile of Prugniel \& Simien (1997) and Lima Neto et al. (1999) fails to reproduce the inner density profiles for low $n$, even up to $n=2.25$ despite the power-law behavior expected at small radii for $n>1$ (Baes \& Gentile 2011).

With a tenth-order two-dimensional polynomial fit, we propose a new analytical approximation (Eq. (28)) that is precise over the range $\log 0.5 \leq \log n \leq 1$, for $-3 \leq \log \left(r / R_{\mathrm{e}}\right) \leq 3$. Our approximation provides the highest precision when averaging over all values of Sérsic indices and radii (Table 1). While the approximations of Simonneau \& Prada (1999, 2004) on one hand and of Emsellem \& van de Ven (2008) on the other are more accurate than ours for $n>4.3$ and 3.4, respectively, ours is more accurate at lower Sérsic indices.

This is important for the study of astronomical sources with low Sérsic indexes, such as galaxy bulges, nuclear star clusters, dwarf spheroidal galaxies, and globular clusters. Moreover, our approximation of Eq. (28) to the density profile is sufficiently accurate for most scientific analyses for $n>3$. Nevertheless, the user could use a hybrid approximation, combining either the Simonneau \& Prada (2004) or Emsellem \& van de Ven (2008) approximations for $n \geq 3.4$ and ours for $n<3.4$ (as shown in the last rows of Table 1). Finally, our analysis has the advantage of also providing a precise approximation for the mass profile, whereas no analytical expression can be derived from the density profile of Emsellem \& van de Ven (2008). Our Python 3 codes are available at ${ }^{4}$ along with coefficients of Tables B.1 and B.2.

These results will be useful in future mass-orbit modeling analyses of low-mass spherical systems, as we are preparing for globular clusters (Vitral \& Mamon, in prep.).

Acknowledgements. We thank Alister Graham for providing important references.

\section{References}

Abel, N. H. 1826, Journal für die reine und angewandte Mathematik, 1, 153 Andredakis, Y. C., Peletier, R. F., \& Balcells, M. 1995, MNRAS, 275, 874 Baes, M., \& Gentile, G. 2011, A\&A, 525, A136

Baes, M., \& van Hese, E. 2011, A\&A, 534, A69

Barmby, P., McLaughlin, D. E., Harris, W. E., Harris, G. L. H., \& Forbes, D. A. 2007, AJ, 133, 2764

Battaglia, G., Tolstoy, E., Helmi, A., et al. 2006, A\&A, 459, 423

Binney, J., \& Mamon, G. A. 1982, MNRAS, 200, 361

Caon, N., Capaccioli, M., \& D'Onofrio, M. 1993, MNRAS, 265, 1013

Ciotti, L., \& Bertin, G. 1999, A\&A, 352, 447

Ciotti, L., Mancino, A., \& Pellegrini, S. 2019, MNRAS, 490, 2656

de Vaucouleurs, G. 1948, Annales d'Astrophysique, 11, 247

Einasto, J. 1965, Trudy Inst. Astroz. Alma-Ata, 51, 87

Emsellem, E., \& van de Ven, G. 2008, ApJ, 674, 653

Graham, A. W., \& Driver, S. P. 2005, PASA, 22, 118

Graham, A. W., \& Spitler, L. R. 2009, MNRAS, 397, 2148

Hernquist, L. 1990, ApJ, 356, 359

Jaffe, W. 1983, MNRAS, 202, 995

Lima Neto, G. B., Gerbal, D., \& Márquez, I. 1999, MNRAS, 309, 481

Łokas, E. L., \& Mamon, G. A. 2001, MNRAS, 321, 155

Márquez, I., Lima Neto, G. B., Capelato, H., Durret, F., \& Gerbal, D. 2000, A\&A, 353, 873

Mazure, A., \& Capelato, H. V. 2002, A\&A, 383, 384

Mellier, Y., \& Mathez, G. 1987, A\&A, 175, 1

Merritt, D., Navarro, J. F., Ludlow, A., \& Jenkins, A. 2005, ApJ, 624, L85

Merritt, D., Graham, A. W., Moore, B., Diemand, J., \& Terzić, B. 2006, AJ, 132, 2685

Muñoz, R. R., Côté, P., Santana, F. A., et al. 2018, ApJ, 860, 66

Navarro, J. F., Frenk, C. S., \& White, S. D. M. 1996, ApJ, 462, 563

Navarro, J. F., Hayashi, E., Power, C., et al. 2004, MNRAS, 349, 1039

Nusser, A. 2019, ArXiv e-prints [arXiv:1907.08035]

Plummer, H. C. 1911, MNRAS, 71, 460

Prugniel, P., \& Simien, F. 1997, A\&A, 321, 111

Sérsic, J. L. 1963, Bull. Assoc. Argent. Astron., 6, 41

Sersic, J. L. 1968, Atlas de Galaxias Australes (Cordoba, Argentina: Observatorio Astronomico)

Simonneau, E., \& Prada, F. 1999, ArXiv e-prints, unpublished

[arXiv:astro-ph/9906151]

Simonneau, E., \& Prada, F. 2004, Rev. Mex. Astron. Astrofis., 40, 69

Trujillo, I., Asensio Ramos, A., Rubiño-Martín, J. A., et al. 2002, MNRAS, 333, 510

Widrow, L. M., \& Dubinski, J. 2005, ApJ, 631, 838 


\section{Appendix A: Comparison of deprojected Sérsic to other popular models}

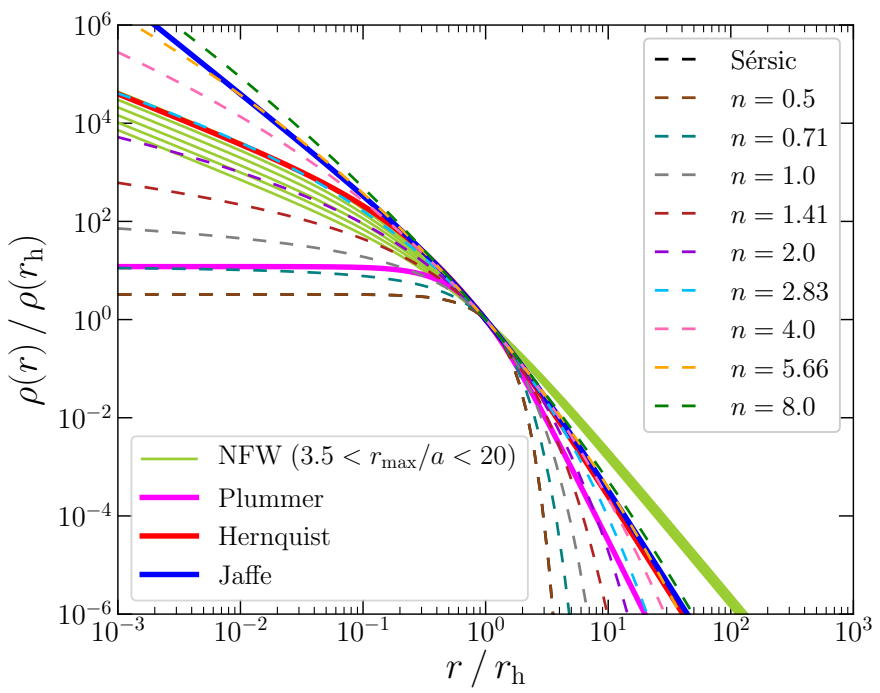

Fig. A.1. Comparison of other known 3D density profiles and the deprojected Sérsic density profile for certain values of the Sérsic index $n$. All density profiles are normalized to the value at the $3 \mathrm{D}$ half-mass radius, $r_{\mathrm{h}}$ (see text). The different NFW models can be distinguished at low radii, where the density increases with $r_{\max } / a$.

Figure A. 1 compares the density profiles, normalized to the halfmass radius $r_{\mathrm{h}}$, for which we applied the following relations:

$\rho(r) \propto \begin{cases}{\left[1+\left(\frac{r}{a}\right)^{2}\right]^{-5 / 2}} & \text { (Plummer), } \\ \left(\frac{r}{a}\right)^{-2}\left[1+\left(\frac{r}{a}\right)\right]^{-2} & (\text { Jaffe }), \\ \left(\frac{r}{a}\right)^{-1}\left[1+\left(\frac{r}{a}\right)\right]^{-3} & \text { (Hernquist), } \\ \left(\frac{r}{a}\right)^{-1}\left[1+\left(\frac{r}{a}\right)\right]^{-2} & \text { (NFW), } \\ \exp \left[-\left(\frac{r}{a}\right)^{1 / n}\right] & \text { (Einasto). }\end{cases}$

The ratio of half-mass radius to scale radius $r_{\mathrm{h}} / a$ is given by

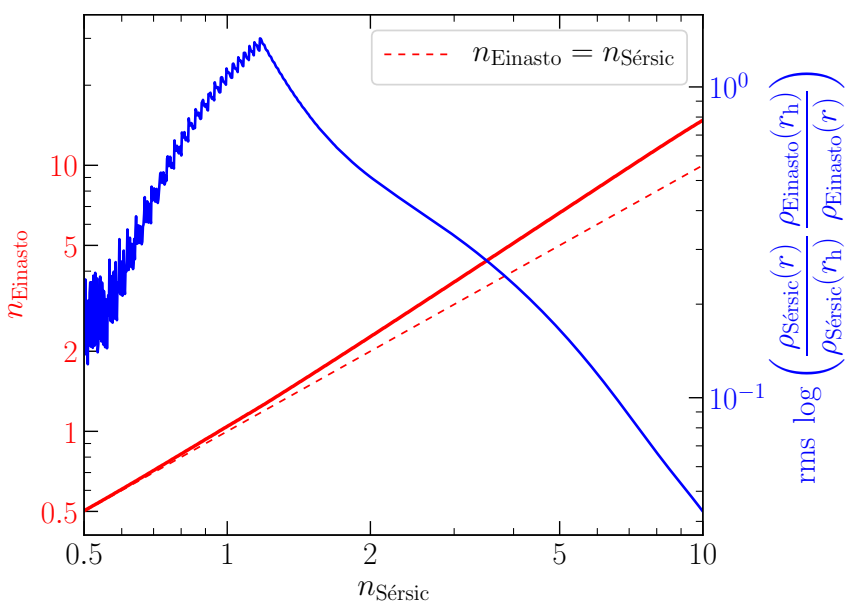

Fig. A.2. Comparison of Einasto and deprojected Sérsic density profiles (both normalized to half-mass radius). Red: best-fit Einasto index (dashed line is $n_{\text {Einasto }}=n_{\text {Sersic }}$ ). Blue: rms of best fit.

$$
\frac{r_{\mathrm{h}}}{a}= \begin{cases}{\left[\left(1+2^{1 / 3}\right) / \sqrt{3}\right]} & (\text { Plummer }), \\ 1 & (\mathrm{Jaffe}), \\ (1+\sqrt{2}) & (\text { Hernquist }), \\ \operatorname{dex}\left(-0.209+0.856 \log c-0.090 \log ^{2} c\right) & (\mathrm{NFW}), \\ {\left[P^{(-1)}(3 n, 1 / 2)\right]^{n}} & (\text { Einasto }) .\end{cases}
$$

In Eq. (A.2) for NFW, $c=r_{\max } / a$, where $r_{\max }$ is the maximum allowed radius (because, contrary to all other models discussed here, the NFW model has logarithmically divergent mass). Also, for Einasto, $P^{(-1)}(a, y)$ is the inverse regularized lower incomplete gamma function, i.e., $x=P^{(-1)}(a, y)$ satisfies $\gamma(a, x) / \Gamma(a)=y^{5}$. For Sérsic, the conversion was done by fitting a third-order polynomial and recovering the relation $r_{\mathrm{h}} / R_{\mathrm{e}}=\sum_{j=0}^{3} a_{i} \log ^{i} n$, where $\left\{a_{0}, a_{1}, a_{2}, a_{3}\right\}=$ $\{1.32491,0.0545396,-0.0286632,0.0035086\}$.

The Einasto model, which is the 3D analog of the Sérsic model, resembles the deprojected Sérsic model. Figure A.2 shows the best-fit values of the Einasto index, $n_{\text {Einasto }}$ in terms of the Sérsic index. The relation (red curve) is almost one-to-one (dashed line). The figure also shows the rms over all radii and best-fit indices (blue curve).

\footnotetext{
5 The inverse (regularized) incomplete gamma function is available in many computer languages, e.g. Python (SCIPY package), Fortran, Matlab, Mathematica, and Javascript.
} 


\section{Appendix B: Coefficients of polynomials for new deprojected Sérsic models}

In this section, we present Tables B.1-B.4, which contain the coefficients $a_{i j}$ in Eq. (26), for $\widetilde{f}=\widetilde{\rho}$ and $\widetilde{f}=\widetilde{M}$, as well as for both Ciotti \& Bertin (1999) approximation for $b_{n}$ and the exact solution from Eq. (2). The numbers in parentheses are the exponents: e.g., "1.234 (-3)" corresponds to $1.234 \times 10^{-3}$. Coefficients that are not followed by a number in parentheses have exponent zero.

Table B.1. Coefficients of Eqs. (26) and (28), for $\widetilde{f}=\widetilde{\rho}$ and $b_{n}$ calculated from Ciotti \& Bertin (1999) accurate approximation.

\begin{tabular}{rrrrrrrrrrrr}
\hline \hline$i \backslash j$ & 0 & \multicolumn{1}{c}{1} & \multicolumn{1}{c}{2} & \multicolumn{1}{c}{3} & \multicolumn{1}{c}{4} & 5 & 6 & 7 & 8 & 9 & 10 \\
\hline 0 & $5.017(-3)$ & $1.573(-3)$ & $-7.175(-2)$ & $1.256(-1)$ & $4.047(-1)$ & -1.191 & $2.455(-1)$ & $8.650(-1)$ & 1.178 & -2.771 & 1.209 \\
1 & $-4.507(-3)$ & $-8.623(-3)$ & $5.430(-2)$ & $2.298(-1)$ & $-9.349(-1)$ & $-3.113(-1)$ & 5.052 & -7.980 & 5.065 & -1.162 & - \\
2 & $-4.251(-2)$ & $6.921(-2)$ & $2.706(-1)$ & -1.134 & 1.318 & $7.078(-1)$ & -3.093 & 2.664 & $-7.604(-1)$ & - & - \\
3 & $1.373(-2)$ & $-2.444(-2)$ & $-8.324(-2)$ & $2.795(-1)$ & $-1.923(-1)$ & $-2.219(-1)$ & $3.670(-1)$ & $-1.381(-1)$ & - & - & - \\
4 & $1.428(-3)$ & $-7.563(-3)$ & $2.897(-4)$ & $5.385(-2)$ & $-1.248(-1)$ & $1.200(-1)$ & $-4.310(-2)$ & - & - & - & - \\
5 & $-1.388(-3)$ & $3.261(-3)$ & $1.615(-3)$ & $-1.112(-2)$ & $1.272(-2)$ & $-5.059(-3)$ & - & - & - & - & - \\
6 & $-9.613(-5)$ & $5.901(-4)$ & $-4.636(-4)$ & $-4.867(-4)$ & $4.043(-4)$ & - & - & - & - & - \\
7 & $9.505(-5)$ & $-1.801(-4)$ & $1.074(-5)$ & $5.734(-5)$ & - & - & - & - & - & - & - \\
8 & $4.306(-6)$ & $-2.496(-5)$ & $2.464(-5)$ & - & - & - & - & - & - & - & - \\
9 & $-2.924(-6)$ & $4.229(-6)$ & - & - & - & - & - & - & - & - & - \\
10 & $-2.117(-8)$ & - & - & - & - & - & - & - & - & - & - \\
\hline
\end{tabular}

Table B.2. Coefficients of Eqs. (26) and (28), for $\widetilde{f}=\widetilde{M}$ and $b_{n}$ calculated from Ciotti \& Bertin (1999) accurate approximation.

\begin{tabular}{rrrrrrrrrrrc}
\hline \hline$i \backslash j$ & 0 & 1 & \multicolumn{1}{c}{2} & \multicolumn{1}{c}{3} & \multicolumn{1}{c}{4} & 5 & 6 & 7 & 8 & 9 & 10 \\
\hline 0 & $7.076(-4)$ & $1.014(-4)$ & $-3.794(-2)$ & $2.785(-2)$ & $4.247(-1)$ & $-6.904(-1)$ & $-6.342(-1)$ & $8.367(-1)$ & 2.565 & -4.115 & 1.622 \\
1 & $1.557(-2)$ & $-4.107(-2)$ & $-6.114(-2)$ & $7.489(-1)$ & -1.700 & $-3.157(-2)$ & 5.508 & -8.413 & 5.055 & -1.080 & - \\
2 & $-3.517(-2)$ & $5.740(-2)$ & $2.328(-1)$ & $-9.638(-1)$ & 1.007 & 1.001 & -3.228 & 2.706 & $-7.776(-1)$ & - & - \\
3 & $1.986(-2)$ & $-2.017(-2)$ & $-1.276(-1)$ & $3.000(-1)$ & $-8.746(-2)$ & $-4.227(-1)$ & $5.202(-1)$ & $-1.817(-1)$ & - & - & - \\
4 & $1.274(-3)$ & $-4.467(-3)$ & $1.989(-3)$ & $3.040(-2)$ & $-7.734(-2)$ & $7.555(-2)$ & $-2.723(-2)$ & - & - & - & - \\
5 & $-2.853(-3)$ & $3.494(-3)$ & $8.720(-3)$ & $-1.959(-2)$ & $1.656(-2)$ & $-6.196(-3)$ & - & - & - & - & - \\
6 & $-1.620(-4)$ & $1.411(-4)$ & $-4.970(-5)$ & $-3.006(-4)$ & $2.779(-4)$ & - & - & - & - & - \\
7 & $2.557(-4)$ & $-3.086(-4)$ & $-2.993(-4)$ & $3.039(-4)$ & - & - & - & - & - & - \\
8 & $1.608(-5)$ & $-2.203(-6)$ & $-3.249(-7)$ & - & - & - & - & - & - & - & - \\
9 & $-9.523(-6)$ & $1.303(-5)$ & - & - & - & - & - & - & - & - & - \\
10 & $-6.227(-7)$ & - & - & - & - & - & - & - & - & - & - \\
\hline
\end{tabular}

Table B.3. Coefficients of Eqs. (26) and (28), for $\widetilde{f}=\widetilde{\rho}$ and $b_{n}$ calculated from Eq. (2).

\begin{tabular}{rrrrrrrrrrrr}
\hline \hline$i \backslash j$ & 0 & 1 & \multicolumn{1}{c}{2} & \multicolumn{1}{c}{3} & 4 & 5 & 6 & 7 & 8 & 9 & 10 \\
\hline 0 & $5.017(-3)$ & $1.573(-3)$ & $-7.176(-2)$ & $1.256(-1)$ & $4.048(-1)$ & -1.191 & $2.460(-1)$ & $8.647(-1)$ & 1.178 & -2.771 & 1.209 \\
1 & $-4.506(-3)$ & $-8.634(-3)$ & $5.434(-2)$ & $2.298(-1)$ & $-9.353(-1)$ & $-3.103(-1)$ & 5.051 & -7.979 & 5.065 & -1.162 & - \\
2 & $-4.251(-2)$ & $6.921(-2)$ & $2.706(-1)$ & -1.134 & 1.318 & $7.077(-1)$ & -3.093 & 2.664 & $-7.605(-1)$ & - & - \\
3 & $1.373(-2)$ & $-2.443(-2)$ & $-8.325(-2)$ & $2.795(-1)$ & $-1.922(-1)$ & $-2.220(-1)$ & $3.670(-1)$ & $-1.381(-1)$ & - & - & - \\
4 & $1.428(-3)$ & $-7.564(-3)$ & $2.912(-4)$ & $5.385(-2)$ & $-1.247(-1)$ & $1.200(-1)$ & $-4.310(-2)$ & - & - & - & - \\
5 & $-1.388(-3)$ & $3.260(-3)$ & $1.617(-3)$ & $-1.112(-2)$ & $1.272(-2)$ & $-5.060(-3)$ & - & - & - & - & - \\
6 & $-9.613(-5)$ & $5.900(-4)$ & $-4.633(-4)$ & $-4.872(-4)$ & $4.045(-4)$ & - & - & - & - & - \\
7 & $9.505(-5)$ & $-1.801(-4)$ & $1.072(-5)$ & $5.735(-5)$ & - & - & - & - & - & - \\
8 & $4.306(-6)$ & $-2.496(-5)$ & $2.464(-5)$ & - & - & - & - & - & - & - & - \\
9 & $-2.924(-6)$ & $4.229(-6)$ & - & - & - & - & - & - & - & - & - \\
10 & $-2.113(-8)$ & - & - & - & - & - & - & - & - & - & - \\
\hline
\end{tabular}

Table B.4. Coefficients of Eqs. (26) and (28), for $\widetilde{f}=\widetilde{M}$ and $b_{n}$ calculated from Eq. (2).

\begin{tabular}{rrrrrrrrrrrr}
\hline \hline$i \backslash j$ & 0 & 1 & \multicolumn{1}{c}{2} & 3 & 4 & 5 & 6 & 7 & 8 & 9 & 10 \\
\hline 0 & $7.075(-4)$ & $1.030(-4)$ & $-3.796(-2)$ & $2.788(-2)$ & $4.249(-1)$ & $-6.912(-1)$ & $-6.335(-1)$ & $8.375(-1)$ & 2.563 & -4.113 & 1.621 \\
1 & $1.557(-2)$ & $-4.107(-2)$ & $-6.112(-2)$ & $7.489(-1)$ & -1.700 & $-3.088(-2)$ & 5.507 & -8.413 & 5.055 & -1.080 & - \\
2 & $-3.518(-2)$ & $5.740(-2)$ & $2.328(-1)$ & $-9.638(-1)$ & 1.007 & 1.001 & -3.228 & 2.706 & $-7.776(-1)$ & - & - \\
3 & $1.986(-2)$ & $-2.017(-2)$ & $-1.276(-1)$ & $3.000(-1)$ & $-8.743(-2)$ & $-4.228(-1)$ & $5.203(-1)$ & $-1.817(-1)$ & - & - & - \\
4 & $1.274(-3)$ & $-4.469(-3)$ & $1.990(-3)$ & $3.040(-2)$ & $-7.734(-2)$ & $7.556(-2)$ & $-2.723(-2)$ & - & - & - & - \\
5 & $-2.853(-3)$ & $3.494(-3)$ & $8.720(-3)$ & $-1.959(-2)$ & $1.656(-2)$ & $-6.195(-3)$ & - & - & - & - & - \\
6 & $-1.620(-4)$ & $1.412(-4)$ & $-4.977(-5)$ & $-3.007(-4)$ & $2.780(-4)$ & - & - & - & - & - \\
7 & $2.557(-4)$ & $-3.086(-4)$ & $-2.993(-4)$ & $3.039(-4)$ & - & - & - & - & - & - & - \\
8 & $1.608(-5)$ & $-2.209(-6)$ & $-3.205(-7)$ & - & - & - & - & - & - & - & - \\
9 & $-9.523(-6)$ & $1.303(-5)$ & - & - & - & - & - & - & - & - & - \\
10 & $-6.228(-7)$ & - & - & - & - & - & - & - & - & - & - \\
\hline
\end{tabular}

